\title{
Geneticists tap human knockouts
}

\author{
Sequenced genomes reveal mutations that disable single genes and can point to new drugs.
}

\section{BY EWEN CALLAWAY}

$\mathrm{F}$ or decades, biologists have studied gene function by inactivating the gene in question in mice and other lab animals, and then observing how it affects the organism. Now researchers studying such gene 'knockouts' have another, ideal model at their disposal: humans.

The approach does not involve genetically engineering mutant people in the lab, as is done in mice. Instead, researchers scan the genomes of thousands or millions of people, looking for naturally occurring mutations that inactivate a particular gene. By observing how these mutations affect health, researchers hope to gain insight into basic biology and to unearth new disease treatments.

Geneticists discussed several such large-scale efforts during a packed session at the American Society of Human Genetics meeting in San Diego, California, last week. "So much of what we know is based on mice and rats, and not humans," says Daniel MacArthur, a genomicist at Massachusetts General Hospital in Boston, whose team identified around 200,000 variations that naturally knocked out genes by trawling the protein-coding portion of the genome, or exome, in more than 90,000 people. "Now we can find people who actually have a particular gene inactivated or somehow modified, and that allows us to test hypotheses directly."

On average, every person carries mutations that inactivate at least one copy of 200 or so genes and both copies of around 20 genes. However, knockout mutations in any particular gene are rare, so very large populations are needed to study their effects. These 'loss of function' mutations have long been implicated in certain debilitating diseases, such as cystic fibrosis. Most, however, seem to be harmless and some are even beneficial to the persons carrying them. "These are people we're not going to find in a clinic, but they're still really informative in biology," says MacArthur.

His group and others had been focusing on genome data, but they are now also starting to mine patient-health records to determine the - sometimes subtle - effects of the mutations. In a study of more than 36,000 Finnish people, published in July (E. T. Lim et al. PLoS Genet. 10, e1004494; 2014), a team that included MacArthur discovered that people lacking a gene called LPA might be protected from heart disease, and that another knockout mutation, carried in one copy of a gene by up to $2.4 \%$ of Finns, may cause fetuses to miscarry if it is present in both copies.

Bing Yu of the University of Texas Health

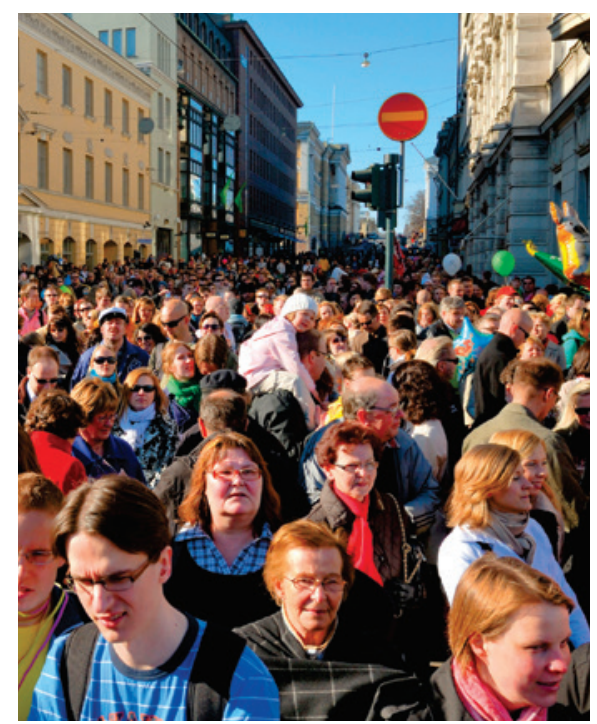

Finland offers geneticists a rich seam of variation.

Science Center in Houston told the meeting how she and her collaborators had compared knockout mutations found in more than 1,300 people with measurements of around 300 molecules in their blood. The team found that mutations in one gene, called SLCO1B1, were linked to high levels of fatty acids, a known risk factor for heart failure. And a team from the Wellcome Trust Sanger Institute in Hinxton, $\mathrm{UK}$, reported that 43 genes whose inactivation is lethal to mice were found to be inactivated in humans who are alive and apparently well.

\section{POSTER CHILD}

Following up on such insights will help researchers to unpick the functions of the thousands of human genes about which little or nothing is known, say MacArthur and others. It might even aid drug discovery by identifying genes or biological pathways that could protect against disease.

The poster child for human-knockout efforts is a new class of drugs that block a gene known as PCSK9 (see Nature 496, 152-155; 2013). The gene was discovered in French families with extremely high cholesterol levels in the early 2000s. But researchers soon found that people with rare mutations that inactivate one copy of PCSK9 have low cholesterol and rarely develop heart disease. The first PCSK9-blocking drugs should hit pharmacies next year, with manufacturers jostling for a share of a market that could reach US $\$ 25$ billion in five years.

"I think there are hundreds more stories like PCSK9 out there, maybe even thousands," in which a drug can mimic an advantageous loss-of-function mutation, says Eric Topol, director of the Scripps Translational Science Institute in La Jolla, California. Mark Gerstein a bioinformatician at Yale University in New Haven, Connecticut, predicts that human knockouts will be especially useful for identifying drugs that treat diseases of ageing. "You could imagine there's a gene that is beneficial to you as a 25-year-old, but the thing is not doing a good job for you when you're 75."

The human-knockout data presented last week will also make it easier to interpret the growing number of genomes being sequenced from people seeking treatment, says Nazneen Rahman, a medical geneticist at the Institute for Cancer Research in London. Rahman told the meeting about her team's analysis of knockout mutations in 1,000 British people. "The take-home message is that these types of mutations occur much more commonly than people thought," she says.

When a person's genome is sequenced to identify the cause of a mysterious illness, any knockout mutations that turn up are obvious suspects. Having a complete list of such mutations and their health effects (or lack thereof) should help to identify the true causes of a disease, Rahman says.

To that end, MacArthur's team last week released knockout and other data from some 63,000 people - and others are already making use of this trove. "One of the first things I did when they released the data was to look at all my favourite genes for very severe diseases, to see if there are people in those databases and there are," says John Belmont, a medical geneticist at Baylor College of Medicine in Houston. He found 11 people with mutations associated with Marfan syndrome, a disorder that affects connective tissue and that can cause heart problems if left untreated.

However, these people may be silent cases, they might somehow be protected against the disease or their genomes might have been sequenced incorrectly. People who carry disease-causing mutations but don't get sick are especially intriguing, he says. "We should pay attention to them," Belmont says. "They may hold a key for new treatments." -

\section{CORRECTION}

The News Feature 'The ethics squad' (Nature 514, 418-420; 2014) misspelled Susan Kornetsky's name. 